\title{
Quimioterapia associada à terapia anti-retroviral de alta eficácia no tratamento dos linfomas não-Hodgkin agressivos relacionados à Síndrome da Imunodeficiência Adquirida \\ Chemotherapy combined with highly active antiretroviral therapy for the treatment of aggressive AIDS related lymphomas
}

\author{
Juliana Pereira ${ }^{1,2}$ \\ Abrahão E Hallack Neto \\ Luís F. Pracchial \\ Andréa Alcântara ${ }^{l}$ \\ Beatriz B. Maurino ${ }^{1,2}$ \\ Pedro E. Dorliac-Llacer ${ }^{l}$ \\ Dalton A. F. Chamone
}

\begin{abstract}
Linfoma não-Hodgkin é uma das complicações oncológicas mais freqüentes em portadores da Síndrome da Imunodeficiência Adquirida (AIDS). Em outros países, após a introdução da terapia anti-retroviral de alta atividade (HAART), a queda na incidência dos linfomas agressivos sistêmicos ficou aquém das expectativas, embora a sobrevida destes pacientes tenha triplicado. No Brasil, pouco se conhece a respeito do comportamento clínico e da sobrevida dos pacientes com linfoma e AIDS na era pós-HAART. O objetivo deste estudo foi avaliar retrospectivamente 25 pacientes com linfoma e AIDS, tratados com a associação de quimioterapia e HAART. Em concordância com a literatura, a maior parte dos pacientes era do sexo masculino - 20 (80\%), com mediana de idade de 39 anos. Houve predomínio do subtipo histológico Difuso de Grandes Células B - 13 (52\%), de pacientes em estádios avançados - 15 (60\%), com envolvimento extranodal - 22 (88\%) e com sintomas B-18 (72\%). O diagnóstico prévio de AIDS observado em 14 (56\%) foi superior em nossa casuística em relação ao descrito por outros autores. Cinqüenta e dois por cento dos pacientes obtiveram RC, com SLD e SG em três anos de 54\% e 42\%, respectivamente e mediana de SG de 15 meses. Toxicidade hematológica e infecções foram freqüentes, porém nenhum óbito foi relacionado à sua ocorrência. Concluímos que o tratamento combinado com quimioterapia e HAART é factível em pacientes brasileiros, podendo propiciar uma sobrevida global similar à descrita por alguns grupos internacionais, com um perfil aceitável de toxicidade. Rev. bras. hematol. hemoter. 2004;26(3):177-182.
\end{abstract}

Palavras-chave: Linfoma; AIDS; terapia anti-retroviral de alta atividade; quimioterapia.

\section{Introdução}

O Brasil é o quarto país em número de casos de Síndrome da Imunodeficiência Adquirida (AIDS). Dados do Ministério da Saúde indicam que até setembro de 2001 havia 227.523 notificações, com declínio da curva de incidência a partir de $1999 .{ }^{1}$ Desde novembro de 1996, a tera- pia anti-retroviral de alta atividade (HAART) tornou-se disponível para todos os pacientes com AIDS e portadores do Vírus da Imunodeficiência Humana (HIV) no Brasil, tendo sido responsável por uma redução de $38 \%$ na mortalidade e de $80 \%$ nos casos de infecções oportunistas associadas a AIDS. ${ }^{2,3}$ Dentre as complicações oncológicas associadas à infecção pelo HIV, o linfoma não-Hodgkin

\footnotetext{
Médico da Disciplina de Hematologia e Hemoterapia da Faculdade de Medicina da Universidade de São Paulo.

${ }^{2}$ Médico do Laboratório de Imunopatologia da Fundação Pró-Sangue Hemocentro de São Paulo.
}

Endereço para correspondência: Juliana Pereira

Laboratório de Imunopatologia, Fundação Pró-Sangue Hemocentro de São Paulo

Avenida Dr. Enéas de Carvalho Aguiar 155, $1^{\circ}$ andar

05403-000 - São Paulo-SP - Brasil

Tel.: (11) 3061-5544 ramal 339 - Fax: (11) 3085-2290 - e-mail: julp2001@ig.com.br 
(LNH) é uma das neoplasias mais freqüentes. Na literatura internacional, não há consenso quanto à redução na incidência de LNH em pacientes HIV positivos em uso de HAART, mas o ganho na sobrevida é indiscutível. ${ }^{4,5}$ Este aumento na sobrevida pode ser explicado pelo maior controle da infecção viral e conseqüente melhora da imunidade ou pelo uso de esquemas quimioterápicos mais agressivos. ${ }^{6,7}$ Em nosso país, entretanto, pouco é sabido sobre a incidência, o comportamento clínico e a sobrevida dos pacientes com linfoma e AIDS na era pós-HAART. ${ }^{8}$ Em nosso serviço, a partir de 1996, passamos a utilizar quimioterapia em baixas doses associada a HAART em pacientes portadores de linfoma e AIDS. Neste estudo apresentamos o resultado de seis anos desta estratégia terapêutica.

\section{Casuística e Métodos}

\section{Pacientes}

Foram incluídos neste estudo retrospectivo todos os pacientes portadores de linfoma não-Hodgkin (LNH) e infecção pelo HIV, confirmada por Western blot, tratados no serviço de Hematologia do Hospital das Clínicas da Faculdade de Medicina da Universidade de São Paulo (HCFMUSP) no período de maio de 1997 a dezembro de 2002. Foram excluídos da análise apenas os pacientes que faleceram em menos de trinta dias do início do tratamento e aqueles com prontuário médico incompleto.

\section{Tratamento}

Todos os casos foram tratados concomitantemente com HAART e quimioterapia. O esquema de HAART utilizado consistia na combinação de dois inibidores da transcriptase reversa (zidovudina, didanosina, estavudina ou lamivudina) e um inibidor de protease (indinavir, nelfinavir ou efanvirens). $O$ tratamento quimioterápico consistiu de seis a oito ciclos de m-BACOD com profilaxia de sistema nervoso central (metotrexate $200 \mathrm{mg} / \mathrm{m}^{2}$ endovenoso D15 bleomicina $4 \mathrm{mg} / \mathrm{m}^{2}$ endovenoso D1, adriamicina $25 \mathrm{mg} / \mathrm{m}^{2}$ endovenoso D1, ciclofosfamida $300 \mathrm{mg} / \mathrm{m}^{2}$ endovenoso D1, vincristina $2 \mathrm{mg}$ endovenoso D1, dexametasona $15 \mathrm{mg} / \mathrm{m}^{2}$ via oral D1 a D5 e dexametasona $2 \mathrm{mg}$ com citarabina $50 \mathrm{mg}$ intratecal $\mathrm{D} 1$, em ciclos de 21 dias) ou seis a oito ciclos de CHOP com profilaxia de sistema nervoso central (ciclofosfamida $750 \mathrm{mg} / \mathrm{m}^{2}$ endovenoso D1, adriamicina $50 \mathrm{mg} / \mathrm{m}^{2}$ endovenoso D1, vincristina 2,0 $\mathrm{mg}$ endovenoso D1, prednisona $60 \mathrm{mg} / \mathrm{m}^{2}$ via oral D1 a D5 e dexametasona $2 \mathrm{mg}$ com citarabina $50 \mathrm{mg}$ intratecal D1, em ciclos de 21 dias). A partir de 2001, os portadores de Linfoma de Burkitt ou Burkitt-atípico foram tratados com quatro ciclos do protocolo experimental para $\mathrm{LNH}$ em pacientes imunossuprimidos ${ }^{9}$ do Instituto Nacional do Câncer dos Estados Unidos da América (NCI) composto por ciclofosfamida $1.200 \mathrm{mg} / \mathrm{m}^{2}$ endovenoso D1 e metotrexate
$3 \mathrm{~g} / \mathrm{m}^{2}$ endovenoso D10, em ciclos de 15 dias. Profilaxia de sistema nervoso central foi realizada com citarabina $60 \mathrm{mg}$ e dexametasona $2 \mathrm{mg}$ intratecal D1, além de citarabina 60 $\mathrm{mg}$, metotrexate $12 \mathrm{mg}$ e dexametasona $2 \mathrm{mg}$ intratecal D10. Filgastrima foi utilizada na dose de $300 \mathrm{mcg} / \mathrm{dia}$ na ocorrência de neutrófilos $<1000 / \mathrm{mm}^{3}$.

Os pacientes foram avaliados ao diagnóstico com hemograma, contagem de células T CD4 em sangue periférico, dosagem de desidrogenase lática sérica (DHL), função renal e hepática, tomografias (cervical, torácica, abdominal e pélvica), exame quimiocitológico do líquido céfaloraquidiano e biópsia de medula óssea bilateral. Os pacientes foram reavaliados após três ou quatro ciclos de quimioterapia, aqueles em remissão completa (RC) receberam um total de seis ciclos e aqueles em remissão parcial (RP) receberam um total de oito ciclos. Os pacientes em estádios I ou II e portadores de lesões bulky ao diagnóstico receberam radioterapia em campo acometido com doses que variaram de 30 a 36 Gy após a obtenção de RC com o tratamento quimioterápico. Os pacientes com diagnóstico de falha da terapia primária, progressão ou recidiva foram tratados com quimioterapia de resgate de forma não padronizada.

\section{Critérios de resposta ao tratamento}

Remissão completa (RC) foi definida como a ausência de todos os sinais e sintomas relacionados ao linfoma por no mínimo quatro semanas após o término do tratamento. Remissão parcial (RP) foi definida como a redução igual ou superior a $50 \%$ da soma dos produtos das duas maiores dimensões perpendiculares das lesões mensuráveis por, no mínimo, quatro semanas na ausência de crescimento de lesões preexistentes, aparecimento de novas lesões ou deterioração das condições clínicas. A redução completa de todos os locais inicialmente comprometidos, porém com persistência de envolvimento de medula óssea foi considerada RP. Qualquer resposta inferior à parcial foi considerada falha da terapia primária. Progressão foi definida como o reaparecimento de lesão prévia, aparecimento de nova lesão ou crescimento igual ou superior a $25 \%$ das lesões residuais. Recidiva foi definida, para os pacientes em RC, como o aparecimento de nova lesão. ${ }^{10}$

\section{Análise estatística}

Revisão de prontuários médicos foi realizada para a obtenção dos seguintes dados: sexo, idade, diagnóstico prévio de AIDS, contagem de células T CD4, tipo histológico do linfoma, sítios acometidos pelo linfoma, estádio de Ann Arbor modificado em Costwolds, ${ }^{11}$ data do início do tratamento, resposta ao tratamento, data da $\mathrm{RC}$, data do óbito, data da recidiva, data do último registro em prontuário, a terapêutica efetuada e suas toxicidades, de acordo com a graduação da Organização Mundial da Saúde 
(OMS). A sobrevida global (SG) foi calculada pelo método de Kaplan-Meier, ${ }^{12}$ considerando-se o período entre o início do tratamento e a data do óbito. A sobrevida livre de doença (SLD), para pacientes em RC, foi calculada da data da RC até a data da recidiva. Os pacientes sem evolução para óbito ou recidiva foram censurados na data do último registro em prontuário. A comparação das taxas de sobrevida estimada em três anos foi realizada pelo teste de Log-rank ${ }^{13}$ bicaudal com erro alfa definido em $5 \%$.

\section{Resultados}

\section{Características dos pacientes}

A mediana de idade dos 25 pacientes incluídos foi de 39 anos, doença extranodal foi verificada em (22) $88 \%$ pacientes e a contagem de células T CD4 inferior a 100 células $/ \mathrm{mm}^{3} \mathrm{em} 32 \%$ dos casos. Infiltração do sistema nervoso central foi encontrada em dois (8\%) pacientes ao diagnóstico.

O principal subtipo histológico foi o Linfoma Difuso de Grandes Células B (LDGCB) em 52\% dos casos e o Linfoma de Burkitt / Burkitt-atípico em 28\% dos pacientes. (Tabela 1).

Tabela 1

Características ao diagnóstico dos 25 pacientes com LNH e HIV

\begin{tabular}{lc}
\hline Características & $\mathrm{n}(\%)$ \\
\hline Mediana de idade (variação) & $39(24-36)$ \\
Sexo masculino & $20(80)$ \\
Diagnóstico prévio de SIDA & \\
Sim & $14(56)$ \\
Não & $11(44)$ \\
Contagem de linfócitos T CD4/mm³ & \\
$\geq 200$ & $11(44)$ \\
$199-100$ & $6(24)$ \\
<100 & $8(32)$ \\
Tipo Histológico & \\
Difuso de Grandes Células B & $13(52)$ \\
Burkitt / Burkitt-atípico & $7(28)$ \\
Células T periféricas & $4(16)$ \\
Plasmablástico & $1(4)$ \\
Estádio Clínico & $10(40)$ \\
I/II & $15(60)$ \\
III / IV & \\
Sítio extranodal & $22(88)$ \\
Presente & $3(12)$ \\
Ausente & \\
DHL & $10(40)$ \\
Normal & $15(60)$ \\
Elevada & \\
Sintomas B & $18(72)$ \\
Presente & $7(28)$ \\
Ausente & $23(8)$ \\
Infiltração de medula óssea & \\
Presente & \\
Ausente & \\
\hline
\end{tabular}

\section{Resultado do tratamento}

Dentre os pacientes avaliados, $19(76 \%)$ foram tratados com m-BACOD, quatro (8\%) pacientes receberam o protocolo experimental do $\mathrm{NCI}^{9}$ e quatro $(8 \%)$ receberam CHOP. Treze (52\%) pacientes obtiveram RC e dois (8\%) RP, resultando em uma taxa de resposta global de 60\%. (Tabela 2)

Com um tempo médio de acompanhamento de 22 meses (variação: 3 a 48 meses) a mediana de SG observada foi de 15 meses. A taxa de SG estimada em três anos foi de $42 \%$ (Figura 1) e a taxa estimada de SLD em três anos foi de 54\% (Figura 2).

Em análise univariada, os tipos histológicos nãoLDGCB, a presença de DHL elevada e a presença de sintomas $\mathrm{B}$ foram os únicos fatores significativamente associados a uma menor taxa de sobrevida global. Alguns fatores não puderam ser avaliados devido ao pequeno número de pacientes disponíveis (Tabela 3).

\section{Toxicidade}

Dezenove pacientes foram avaliáveis para toxicidade relacionada ao tratamento, $16(84 \%)$ desenvolveram neutropenia grau 3 ou 4 em pelo menos um dos ciclos de quimioterapia e 12 (63\%) desenvolveram infecções não

Tabela 2

Resposta terapêutica dos 25 pacientes com LNH e HIV

\begin{tabular}{lc}
\hline \multicolumn{1}{c}{ Resposta } & $\mathrm{n}(\%)$ \\
\hline Remissão completa & $13(52)$ \\
Remissão carcial & $2(8)$ \\
Progressão ou falha & $10(40)$ \\
\hline
\end{tabular}

Figura 1

Curva de Sobrevida Global de 25 pacientes com linfoma sistêmico e AIDS

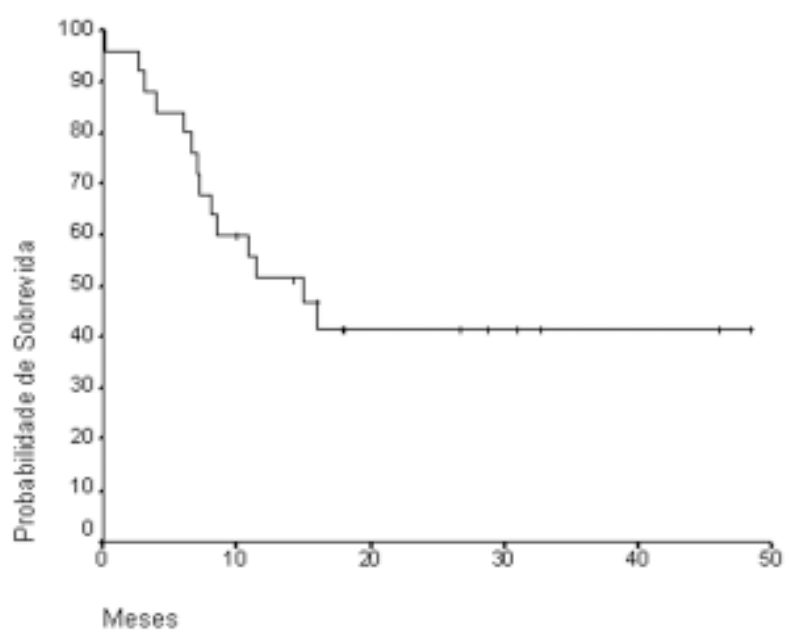

Mediana de SG de 15 meses (IC 95\%= $8-22$ meses) Taxa de Sobrevida Global em 3 anos de $42 \%$ 
oportunistas de qualquer grau (Tabela 4). Neurotoxicidade grau 3 ou 4 foi evidenciada em quatro $(21 \%)$ pacientes, todos evoluíram para resolução completa do quadro após o término da quimioterapia.

Figura 2

Curva de Sobrevida Livre de Doença de 25 pacientes com linfoma sistêmico e AIDS

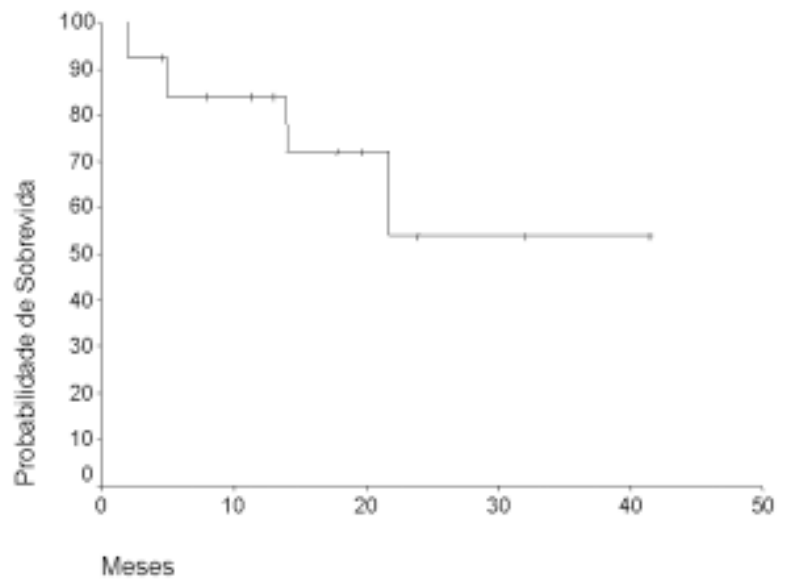

Mediana de SLD não atingida.

Média de SLD de 29 meses (IC 95\%= 19 - 38 meses).

Taxa de Sobrevida Livre de Doença em 3 anos de 54\%

Tabela 3

Análise univariada de fatores prognósticos e Sobrevida Global

\begin{tabular}{|c|c|c|}
\hline Característica ao diagnóstico & $\begin{array}{c}\text { SG } 3 \text { anos } \\
(\%)\end{array}$ & $\mathrm{P}$ \\
\hline \multicolumn{3}{|l|}{ Sexo } \\
\hline Masculino & 39 & 0,660 \\
\hline Feminino & 55 & \\
\hline \multicolumn{3}{|l|}{ Diagnóstico prévio de AIDS } \\
\hline Sim & 37 & 0,570 \\
\hline Não & 46 & \\
\hline \multicolumn{3}{|c|}{ Contagem de linfócitos $T \mathrm{CD} 4 / \mathrm{mm}^{3}$} \\
\hline$\geq 200$ & 53 & 0,760 \\
\hline$<199$ & 31 & \\
\hline \multicolumn{3}{|l|}{ Tipo Histológico } \\
\hline LDGCB & 57 & 0,077 \\
\hline Não-LDGCB & 17 & \\
\hline \multicolumn{3}{|l|}{ Estádio Clínico } \\
\hline $\mathrm{I} / \mathrm{II}$ & 59 & 0,211 \\
\hline III / IV & 29 & \\
\hline \multicolumn{3}{|l|}{$D H L$} \\
\hline Até o normal & 69 & 0,023 \\
\hline Acima do normal & 21 & \\
\hline \multicolumn{3}{|l|}{ Sintomas $B$} \\
\hline Presentes & 32 & 0,041 \\
\hline Ausentes & 69 & \\
\hline \multicolumn{3}{|l|}{ Tratamento } \\
\hline m-BACOD & 45 & 0,621 \\
\hline $\mathrm{CHOP}$ ou Protocolo $\mathrm{NCl}^{9}$ & 30 & \\
\hline
\end{tabular}

Tabela 4

Toxicidade do tratamento dos 19 pacientes com LNH e HIV

\begin{tabular}{lc}
\hline Tipo de toxicidade & $\begin{array}{c}\text { Freqüência } \\
\mathrm{n}(\%)\end{array}$ \\
\hline Neutropenia & $16(84)$ \\
Plaquetopenia & $1(5)$ \\
Anemia & $1(5)$ \\
Mucosite & $1(5)$ \\
Infecciosa & $12(63)$ \\
Neurológica & $4(21)$ \\
\hline
\end{tabular}

Nota: Considerou-se pelo menos um episódio de toxicidade hematológica, тисоsa ou neurológica grau 3 ou 4 pela OMS ocorrida em qualquer ciclo e pelo menos um episódio de infecção de qualquer grau OMS ocorrida em qualquer ciclo.

\section{Discussão}

No presente estudo descrevemos as características clínicas e resposta ao tratamento de 25 pacientes não selecionados portadores de linfoma agressivo e AIDS, referenciados e tratados de forma consecutiva em nossa instituição. Embora o HC-FMUSP seja uma instituição de referência para tratamento de casos complexos de todo o Brasil, o que poderia levar a uma seleção involuntária da população estudada, nós observamos, assim como autores internacionais, ${ }^{4,5,7}$ um predomínio de pacientes do sexo masculino (80\%), de linfomas de fenótipo B agressivos em $80 \%$ dos casos e do subtipo Difuso de Grandes Células B (52\%). A maior parte dos pacientes apresentava doença avançada ao diagnóstico (estádio III e IV), comprometimento extranodal, sintomas B, DHL elevada e dosagem de CD4 acima de 100 células $/ \mathrm{mm}^{3}$. Como referido na literatura, encontramos alto índice de pacientes com diagnóstico de AIDS antes do LNH. ${ }^{14} \mathrm{~A}$ taxa de RC (52\%), a mediana de SG (15 meses) e a toxicidade foram semelhantes às observadas em estudos que utilizaram terapêutica similar à nossa ${ }^{15,18} \mathrm{e}$ superiores às verificadas na era préHAART. ${ }^{20}$ Porém, as características clínicas ao diagnóstico, incluindo idade, sexo, histologia, proporção de indivíduos com DHL elevada, infiltração de medula óssea e sítios extranodais foram semelhantes aos casos préHAART. ${ }^{15,20}$

A não existência de consenso quanto ao tratamento de pacientes com LNH e AIDS incentivou a investigação de novos protocolos visando aumentar a sobrevida destes pacientes. Após a introdução da HAART e conseqüente redução da morbidade dos pacientes, muitos pesquisadores começaram a utilizar tratamentos mais agressivos. Alguns optaram pelo uso de altas doses de quimioterapia ${ }^{16}$ e outros pela infusão contínua de drogas. Com esta última estratégia, Sparano ${ }^{17}$ obteve RC de $67 \%$, superior à encontrada em nosso estudo, mas a SG de 17,4 meses foi semelhante à nossa. Entretanto, neste estudo 
apenas um paciente tinha critério de AIDS antes do linfoma. Observamos taxas de RC, SLD e SG inferiores às obtidas com o protocolo de infusão contínua $\mathrm{EPOCH},{ }^{7}$ porém, neste estudo, foram incluídos apenas pacientes sem diagnóstico prévio de AIDS. Em nossa casuística, 56\% dos pacientes tinham diagnóstico de AIDS antes de desenvolverem linfoma. Apesar de não termos demonstrado, a SG é inferior em indivíduos com critério de AIDS antes do linfoma e está associada à eficácia da terapia anti-retroviral. ${ }^{5} \mathrm{Em}$ análise univariada, a presença de sintomas B e o aumento de DHL foram fatores estatisticamente significantes para a redução da SG.

O tipo histológico do linfoma também teve importância prognóstica em nossa série. A SG dos pacientes com LDGCB (57\%) superou a obtida em pacientes com Linfoma de Burkitt e células T (17\%). Quando observamos este fato, passamos a tratar os pacientes com Linfoma de Burkitt com ciclofosfamida e metotrexate em altas doses. ${ }^{9}$ Com esta estratégia estamos conseguindo melhorar nossos resultados com toxicidade aceitável (dados não apresentados). O único paciente com linfoma plasmablástico apresentou-se com lesão em seios da face e obteve excelente resposta com quimioterapia (m-BACOD) e radioterapia.

Neutropenia, infecção e neurotoxicidade foram as principais toxicidades encontradas. Os pacientes receberam fator de crescimento quando apresentavam neutrófilos inferiores a $1000 / \mathrm{mm}^{3}$ e nenhum paciente foi a óbito em decorrência de infecção. Quatro (21\%) pacientes apresentaram neuropatia periférica reversível, fato que pode ser explicado por possíveis interações entre os quimioterápicos e as drogas anti-retrovirais. Os inibidores de protease e os inibidores da transcriptase reversa não nucleosídeos podem interferir no metabolismo da ciclofosfamida, alcalóides da vinca, etoposide e antraciclinas, potencializando os efeitos colaterais destas drogas. ${ }^{18}$ Além disso, os nucleosídeos inibidores de transcriptase reversa podem, isoladamente, causar neuropatia periférica ${ }^{19}$ e seu uso concomitante com vincristina e metotrexate pode potencializar este efeito.

Tanto as evidências internacionais acima expostas quanto os resultados do presente estudo demonstram que, após a introdução da HAART, houve significativa melhora na sobrevida dos pacientes com linfoma e AIDS. Porém, o melhor esquema de tratamento ainda não foi estabelecido. A criação de grupos cooperativos brasileiros para a realização de estudos randomizados será necessária para esclarecer esta questão em nosso país.

\section{Abstract}

Non-Hodgkin lymphoma is one of the most frequent oncological complications in patients with the Acquired Immune-Deficiency Syndrome (AIDS). In other countries, after the introduction of the Highly Active Antiretroviral Therapy (HAART), the drop in the incidence of systemic aggressive lymphomas was below expectations, although the survival of these patients rose. In Brazil, little is known about the clinical behavior and survival of the patients with lymphoma and AIDS in the post-HAART era. The aim of this study was to retrospectively evaluate 25 patients with lymphomas and AIDS, treated with the combination of chemotherapy and HAART. In agreement with the literature most of the patients were male (20 patients - 80\%) with a median age of 39 years. We observed a predominance of the Diffuse Large B Cell Lymphoma subtype (13 patients - 52\%), advanced stage (15 patients - 60\%), with extra-nodal disease (22 patients - $88 \%$ ) and B symptoms (18 patients - 72\%). Previous AIDS diagnosis was present in 14 patients (56\%), higher than that reported in other series. Fifty-two percent achieved $C R$, the estimated probability of overall survival and disease-free survival at 3 years were $54 \%$ e $42 \%$, respectively. The median overall survival time was 15 months. Hematological toxicity and infections were frequently observed, but no toxicity-related deaths were seen. Therefore we conclude that the combined chemotherapy-HAART treatment is feasible in Brazilian patients and can provide similar overall survival than that described for some international groups, with an acceptable toxicity profile. Rev. bras. hematol. hemoter. 2004;26(3):177-182.

Key words: Lymphoma; AIDS; Highly active antiretroviral therapy; chemotherapy.

\section{Referências Bibliográficas}

1. Boletim epidemiológico (AIDS) da Coordenação Nacional de DST e

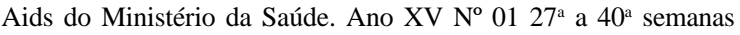
epidemiológicas Julho a Setembro de 2001 - Editorial.

2. Marins JRP, Jamal LF, Chen SY et al. Dramatic improvement in survival among adult Brazilian AIDS patients. AIDS 2003;17: 1.675-82.

3. Nobre V, Braga E, Rayes A et al. Infecções oportunistas em pacientes com AIDS internados em um hospital universitário do sudeste do Brasil. Rev Inst Med Trop S Paulo 2003; $45: 274-8$.

4. Otieno MW, Banura C, Katongole-Mbidde E et al. Therapeutic Challenges of AIDS-Related Non-Hodgkin's Lymphoma in the United States and East Africa - A review. J Natl Cancer Inst 2002;94:718-32.

5. Hoffman C, Wolfi E, Fätkenheuer G et al. Response to highly active antiretroviral therapy strongly predicts outcome in patients with AIDS-related lymphoma. AIDS 2003;17:1.52129.

6. Tirelli U, Spina M, Jaeger U et al. Infusional CDE with rituximab for the treatment of human immunodeficiency virus-associated non-Hodgkin lymphoma: preliminary results of a phase I/II study. Recent Results Cancer Res 2002;159:149-53.

7. Little RF. Highly effective treatment of acquired Immunodeficiency Syndrome-Related Lymphoma with dose adjusted EPOCH: impact of antiretroviral therapy suspension and tumor biology. Blood 2003;101:4.653-9.

8. Baiocchi OC, Colleoni GW, Navajas EV et al. Impact of highly active antiretroviral therapy in the treatment of HIV-infected patients with systemic non-Hodgkin's lymphoma. Acta Oncol 2002;41:192-6. 
9. Magrath IT, Shad AT, Sandlund JT. Lymphoproliferative disorders in immunocompromised individuals. In The NonHodgkin's Lymphomas. Ed. Ian Magrath, second edition. Chapter 45, p.955-74. London, Great Britain, 1997.

10. Cheson BD, Horning SJ, Coiffier B et al. Report of an International Workshop to Standardize Response Criteria for Non-Hodgkin's Lymphomas. J Clin Oncol 1999;17:1.2441.253 .

11. Carbone PP, Kaplan HS, Musshoff $\mathrm{K}$ et al. Report of the Committee on Hodgkin's Disease Staging Classification. Cancer Res 1971; 31:1.860-1.

12. Kaplan EL, Meier P. Nonparametric estimation from incomplete observations. J Am Stat Assoc 1958;53:457-81.

13. Mantel, N. Evaluation of survival data and two new rank order statistics arising in its consideration. Cancer Chemother Rep 1966;50:163-70.

14. Knowles DM, Chamulak GA, Subar M. Lymphoid neoplasia associated with the acquired immunodeficiency syndrome (AIDS). The New York University Medical Center experience with 105 patients (19811986). Ann Intern Med 1988;5:744-53.

15. Besson C, Goubar A, Gabarre J et al. Changes in AIDS-related lymphoma since the era of highly active antiretroviral therapy. Blood 2001;98:2.339-44.

16. Wang ES, Straus DJ, Teruya-Feldstein $J$ et al. Intensive chemotherapy with cyclophosphamide, doxorubicin, high-dose methotrexate/ifosfamide, etoposide, and high-dose cytarabine (CODOX-M/IVAC) for human immunodeficiency virusassociated Burkitt lymphoma. Cancer 2003;98:1.196-205.

17. Sparano JA, Wiernik PH, Leaf A et al. Infusional cyclophosphamide, doxorubicin, and etoposide in Human Immunodeficiency Virus - and Human T-cell leukemia virus type I-related non-Hodgkin lymphoma: A highly active regimen. Blood 81:2.810-5.

18. Levine AM, Tulpule A, Espina B et al. Low dose methotrexate, bleomycin, doxorubicin, cyclophosphamide, vincristine, and dexamethasone with zalcitabine in patients with acquired immunodeficiency syndrome-related lymphoma. Effect on human immunodeficiency virus and serum interleukin-6 levels over time. Cancer 1996;78:517-26.

19. Dragovic G, Jevtovic D. Nucleoside reverse transcriptase inhibitor usage and the incidence of peripheral neuropathy in HIV/AIDS patients. Antivir Chem Chemother 2003;14:281-4.

20. Kaplan, Lawrence D et al: Low-dose compared with standarddose m-BACOD chemotherapy for non-Hodgkin's lymphoma associated wiyh Human Immunodeficiency Virus Infection. NEJM 1997;336:1.641-8

Avaliação: Editor e dois revisores externos.

Conflito de interesse: não declarado

Recebido: 25/05/2004

Aceito após modificações: 23/08/2004 